\title{
Primary pulmonary artery Rosai-Dorfman disease mimicking sarcoma
}

\author{
Marco Morsolini, MD, PhD, ${ }^{\mathrm{a}}$ Marta Nicola, MD,${ }^{\mathrm{b}}$ Marco Paulli, MD, PhD,${ }^{\mathrm{b}}$ and \\ Andrea M. D'Armini, MD, ${ }^{\mathrm{a}}$ Pavia, Italy
}

Rosai-Dorfman disease (RDD) is a rare disorder in which the nodal sinuses are engulfed by large, eosinophilic histiocytes containing intact lymphocytes within their cytoplasm (emperipolesis, the diagnostic hallmark). ${ }^{1}$ Despite its reactive, non-neoplastic nature, the etiology remains a matter of debate. ${ }^{2}$

The typical presentation includes painless selfregressing lymphadenopathy, with involvement of extranodal sites in up to $43 \%$ of cases. Primary extranodal RDD occurs in $23 \%$ of cases, with the skin, soft tissue, and upper respiratory tract the most frequently affected sites. ${ }^{1}$ $\mathrm{RDD}$ is a benign condition; however, the involvement of vital organs can be associated with a potentially fatal outcome. Cardiovascular localization is life-threatening and associated with chest pain, severe dyspnea, and heart failure. ${ }^{3-5}$

We describe the surgical treatment of a case of primary extranodal RDD involving the pulmonary artery (PA) and the apex of the heart.

\section{CASE REPORT}

A 62-year-old man with a history of resected ileal neuroendocrine tumor and right hepatectomy for metastasis, who was disease free at 6 years of follow-up surveillance, presented in 2012 with a dry cough. Computed tomography revealed a $23-\mathrm{mm}$ mass infiltrating the right PA, causing significant stenosis and showing high metabolic activity with 2-deoxy-2[18F]fluoro-D-glucose positron emission tomography. The patient was referred to our institution for operability assessment. A total body computed tomography scan confirmed the presence

\footnotetext{
From the Division of Cardiac Surgery ${ }^{\mathrm{a}}$ and Anatomic Pathology Section, ${ }^{\mathrm{b}}$ University of Pavia School of Medicine, Foundation I.R.C.C.S. Policlinico San Matteo, Pavia, Italy.

The present work was supported by an Investigator Fellowship from Collegio Ghislieri, Pavia, Italy.

Disclosures: Authors have nothing to disclose with regard to commercial support.

Received for publication June 5, 2013; revisions received July 17, 2013; accepted for publication Aug 1, 2013; available ahead of print Sept 30, 2013.

Address for reprints: Andrea M. D'Armini, MD, Division of Cardiac Surgery, University of Pavia School of Medicine, Foundation I.R.C.C.S. Policlinico San Matteo, Viale Camillo Golgi, 19, Pavia 27100, Italy (E-mail: darmini@ smatteo.pv.it).

J Thorac Cardiovasc Surg 2013;146:e57-9

$0022-5223 / \$ 36.00$

Copyright (c) 2013 by The American Association for Thoracic Surgery

http://dx.doi.org/10.1016/j.jtcvs.2013.08.001
}

of a highly vascularized lesion (40 $\mathrm{mm}$ in length), stemming from the right lateral wall of the pulmonary trunk, $10 \mathrm{~mm}$ beyond the pulmonary valve, and extending toward the origin of the right upper lobe artery (Figure 1, A). Transmural infiltration was highly suspected. No other pathologic findings, such as abdominal recurrence or carcinoid heart disease, were detected at computed tomography.

From his medical history and preoperative findings, a PA sarcoma was suspected, and the patient underwent surgery (Figure 1, B). An endovascular biopsy was excluded because of the high risk of embolization. The mass was removed, leaving the pulmonary wall macroscopically free of disease on intraoperative inspection (Figure 1,C). A surgical specimen was sent to the pathology laboratory for intraoperative examination, which ruled out sarcoma but showed a margin-free polymorphous inflammatory cellular infiltrate. Therefore, unilateral pulmonary endarterectomy of the right main PA was completed. A thin, superficial, whitish plaque was observed, covering the apex of the heart, neither infiltrating nor affecting contractility. Considering the benign nature and location, it was not removed.

The remaining tissue was fixed in formalin and embedded in paraffin. Examination revealed a background population of mature plasma cells; small lymphocytes; neutrophils; foamy histiocytes with a xanthogranulomatous appearance; and numerous large histiocyte-like cells with loose chromatin and a wide, eosinophilic cytoplasm containing intact lymphocytes, neutrophils, plasma cells, and erythrocytes (Figure 1, $D$ and $E$ ) that stained intensely for S-100 protein (Figure 1, F), CD68R/PGM1, CD163, CD14, and FXIIIa and faintly for CD1a (cytoplasmic positivity). Immunostains for the kappa and lambda light chains revealed polyclonal plasma cells, and IgG4 highlighted rare positive elements. The growth rate, evaluated using Mib-1/Ki-67 antibody, was very low $(1 \%)$. With these findings, extranodal RDD was diagnosed.

The patient's postoperative course was fast and uneventful. No adverse events or complications occurred. No medical therapy was administered, and a wait-and-see approach with magnetic resonance imaging was adopted for the PA and apex of the heart. At 9 months of followup, the patient was alive, disease free, and in good clinical condition. 

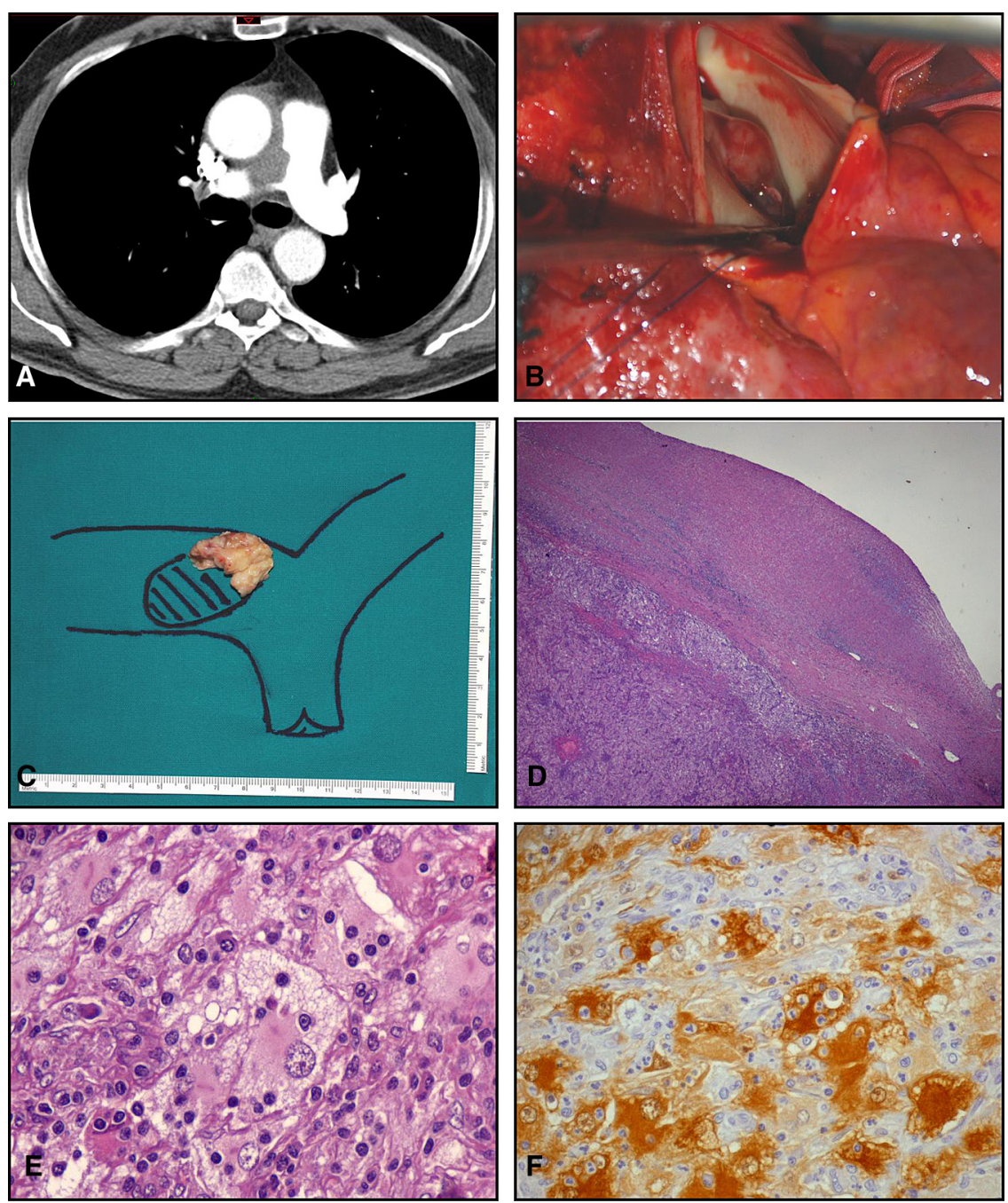

FIGURE 1. A, Computed tomography scan showing the solid mass obliterating the lumen of the right PA. B, Intraoperative visualization of the solid mass. $\mathrm{C}$, Gross appearance of the removed mass showing white-yellow to tan solid fleshy tissue. D, Microscopic appearance, with background composed of mature plasma cells, small lymphocytes, foamy histiocytes, and large histiocyte-like cells (hematoxylin-eosin stain, original magnification $\times 2$ ). E, Intact lymphocytes within the cytoplasm of large histiocyte-like cells (emperipolesis; hematoxylin-eosin stain, original magnification $\times 60$ ). F, Histiocyte-like cells showing intense positivity for S-100 protein (streptavidin-biotin complex method, original magnification $\times 40$ ).

The institutional ethical committee approved the present study, and data management was performed in accordance with the Declaration of Helsinki.

\section{DISCUSSION}

The diagnosis of RDD is seldom considered because of its rarity and low index of suspicion. Although the patient had neither nodal nor other extranodal localization, an early diagnosis was promptly established before the development of heart failure, because of the increased clinical surveillance related to his medical history and the referral to our institution for operability assessment with the suspicion of PA sarcoma.

In the present unusual extranodal case of RDD, the differential diagnoses included inflammatory myofibroblastic tumor, vasculitis, inflammatory pseudotumor-like follicular dendritic-cell sarcoma, and metastatic melanoma. Moreover, the possibility of metastasis from the previous neuroendocrine tumor and the clinical suspicion of intimal sarcoma had to be ruled out.

We report the third case of extranodal RDD involving the PA. A cardiovascular presentation can be challenging for cardiovascular surgeons, because it closely mimics primary PA sarcoma and requires a specific surgical approach. Surgical debulking was mandatory because of the lifethreatening localization. ${ }^{3-5}$ Unilateral pneumonectomy seems more suitable to obtain oncologic radicality in the case of unilateral PA sarcoma, whereas a conservative procedure using pulmonary endarterectomy is indicated in the case of RDD. Therefore, an intraoperative histologic examination is crucial to plan the most appropriate 
surgical treatment. Finally, pathologists must be aware that RDD should be considered among the possible differential diagnoses for cardiovascular tumors.

\section{References}

1. Foucar E, Rosai J, Dorfman R. Sinus histiocytosis with massive lymphadenopathy (Rosai-Dorfman disease): review of the entity. Semin Diagn Pathol. 1990;7:19-73.

2. Paulli M, Bergamaschi G, Tonon L, Viglio A, Rosso R, Facchetti F, et al. Evidence of a polyclonal nature of the cell infiltrate in sinus histiocytosis with massive lymphadenopathy (Rosai-Dorfman disease). Br J Haematol. 1995;91: 415-8.

3. Walters DM, Dunnington GH, Dustin SM, Frierson HF, Peeler BB, Kozower BD, et al. Rosai-Dorfman disease presenting as a pulmonary artery mass. Ann Thorac Surg. 2010;89:300-2.

4. Ajise OE, Stahl-Herz J, Goozner B, Cassai N, McRae G, Wieczorek R. Extranodal Rosai-Dorfman disease arising in the right atrium: a case report with literature review. Int J Surg Pathol. 2011;19:637-42.

5. Prendes BL, Brinkman WT, Sengupta AL, Bavaria JE. Atypical presentation of extranodal Rosai-Dorfman disease. Ann Thorac Surg. 2009;87: 616-8.

\title{
Pseudo-dextrocardia: An unusual case of giant biatrial enlargement
}

\author{
Roberto Lorusso, MD, PhD, ${ }^{\mathrm{a}}$ Mario Morone, MD, ${ }^{\mathrm{b}}$ Davide Farina, $\mathrm{MD},{ }^{\mathrm{b}}$ and \\ Sandro Gelsomino, $\mathrm{MD}, \mathrm{PhD},{ }^{\mathrm{a}}$ Brescia, Italy
}

Giant dilatation of cardiac atria is usually confined to 1 chamber, very rarely to both, and is mainly related to rheumatic valve diseases of the left heart. ${ }^{1-3}$ Cases of left atrial enlargement have also been reported with a normally functioning mitral or aortic valve and extremely rarely in the presence of nonrheumatic mitral insufficiency., ${ }^{2,3}$ The combination of giant bilateral atrial dilatation and the need of reoperation for cardiac prosthetic dysfunction can pose additional risks because of adhesions and a thin right atrial wall. Furthermore, the unusual cardiac displacement induced by excessive atrial enlargement can jeopardize, or even impede, conventional access to the major vessels for extracorporeal circulation (ECC).

We report the case of a patient who had presented with a "pseudo-dextrocardia" found by chest radiography. The pseudo-dextrocardia was induced by an unusual bilateral atrial dilatation. The patient required a third operation for a paravalvular leak of a mitral prosthesis.

\section{CLINICAL REPORT}

A 62-year-old man was referred to our hospital for increasing dyspnea. At 25 years old, he had undergone mitral commissurotomy; 12 years later, he had undergone mitral-aortic replacement and tricuspid valve repair for rheumatic-related valve disease. Marked bilateral

\footnotetext{
From the Cardiac Surgery Unit ${ }^{\mathrm{a}}$ and Radiology Unit, ${ }^{\mathrm{b}}$ Community Hospital, Brescia, Italy.

Disclosures: Authors have nothing to disclose with regard to commercial support.

Received for publication June 6, 2013; revisions received July 20, 2013; accepted for publication Aug 1, 2013; available ahead of print Sept 23, 2013.

Address for reprints: Roberto Lorusso, MD, PhD, Cardiac Surgery Unit, Community Hospital, Piazzale Spedali Civili, 1, Brescia 25128, Italy (E-mail: ro.lorusso@ libero.it).

J Thorac Cardiovasc Surg 2013;146:e59-61

$0022-5223 / \$ 36.00$

Copyright (C) 2013 by The American Association for Thoracic Surgery

http://dx.doi.org/10.1016/j.jtcvs.2013.08.014
}

atriomegaly had been present since the first surgical procedure because of severe mitral stenosis with moderate tricuspid regurgitation, which had not been addressed during that operation. Additional progression of the atrial dilatation had developed owing to the recurrence of severe mitral stenosis, the development of severe aortic stenosis, and the development of tricuspid insufficiency requiring reoperation. At that point, because of the tight adhesions between the right ventricle and right atrium and the sternum, making sternal reentry troublesome, a right anterior minithoracotomy had been performed to enhance adhesion dissection and allow safer median sternotomy. Severe bleeding had nonetheless occurred, although it was finally controlled, allowing a triplevalve operation. The patient subsequently did well until a few months before his latest hospitalization for shortness of breath. At hospital admission, the chest radiograph showed a huge cardiac enlargement, mimicking a dextrocardia, and diffuse adhesions between the right cardiac structures and the sternum (Figure 1). The anatomic features were confirmed by computed tomography (Figure 2). Severe hemolysis was also detected from the blood test results, with marked anemia and initial signs of liver dysfunction. Transesophageal echocardiography showed severe mitral regurgitation due to a large posteromedial paravalvular leak, normal function of the aortic mechanical prosthesis and tricuspid valve, and a very short ascending aorta, making direct access for cannulation or the application of an endovascular aortic clamping unfeasible or extremely dangerous. A right thoracotomy approach and femoral-femoral ECC was therefore scheduled. The patient was cooled down to $24^{\circ} \mathrm{C}$ to induce ventricular fibrillation and allow a short period of circulatory arrest, avoiding aortic clamping. The left atrium was approached with extreme difficulty after dissection of dense adhesions 